\title{
Adsorption of a macromolecule to a charged surface
}

\author{
F W Wiegel \\ Department of Applied Physics, Twente University of Technology, PO Box 217, Enschede, \\ The Netherlands
}

Received 21 July 1976, in final form 24 September 1976

\begin{abstract}
We investigate the adsorption of a charged macromolecule to a charged surface under the influence of a weak electrostatic attraction. The probability density $P(x, N)$ to find the end point of a molecule with $N$ monomer units at distance $x$ from the surface is calculated analytically. In the limit $N \rightarrow \infty$ this function shows a phase transition at a critical value $\left(\theta_{\mathrm{c}}\right)$ of the adsorption energy in units of $k_{\mathrm{B}} T$ : for $\theta>\theta_{\mathrm{c}} P(x, N)$ is peaked near the surface and the molecule is effectively adsorbed to the surface; for $\theta<\theta_{c}$ the heat motion is strong enough to remove the molecule from the surface and $P(x, N)$ is effectively constant throughout the whole available volume. We comment briefly upon a possible role of such conformational phase transitions in the regulation of cellular metabolism.
\end{abstract}

\section{Introduction}

The adsorption of a macromolecule to a surface has been studied by several authors. Of the more recent papers we should like to quote the study by Rubin (1965), who restricted the chain to the bonds of a lattice and who obtained analytic expressions for various statistical quantities of interest. In Rubin's model the attractive forces between the molecule and the surface have a range which is comparable to the size of a monomer unit. The most interesting aspect of the analytic solutions which this author found is the existence of a discontinuity in the adsorption behaviour at some critical value $\left(\theta_{c}\right)$ of the adsorption energy in units $k_{\mathrm{B}} T$ : for $\theta>\theta_{\mathrm{c}}$ the macromolecule exists in an adsorbed state; for $\theta<\theta_{\mathrm{c}}$ the heat motion is strong enough to tear it off the surface and shuttle it throughout the whole fluid.

These results are all the more interesting because Di Marzio and Bishop (1974) showed that the presence of a surface sharpens the helix-coil transition both in polypeptides and in polynucleotides.

In view of the unknown character of the forces which act in the living cell between biopolymers and cell membranes it is of interest to study a model for adsorption which is the opposite of Rubin's model, in that the attractive force is long-ranged rather than short-ranged. As a candidate for such interactions we consider in this paper the case that the macromolecule carries a fixed charge on each repeating unit, and that the membrane carries fixed charges on its surface with a constant surface charge density. The electrostatic forces between the repeating units of the macromolecule will be neglected. For a weakly bound, very long macromolecule the adsorption problem can be solved in detail, as will be shown in $\$ 2$. 


\section{Theory}

If the charge per repeating unit of the macromolecule is denoted by $q$ and the surface charge density of the membrane by $\sigma$, the energy of the electrostatic interaction between a repeating unit and a surface element of area $\mathrm{d}^{2} S$ equals:

$$
\mathrm{d}^{2} V=(q \sigma / \epsilon r) \exp (-k r) \mathrm{d}^{2} S .
$$

Here $\epsilon$ denotes the dielectric constant of the cell fluid and $k^{-1}$ is the Debye screening length, a measure for the distance beyond which the ions in the cell fluid have screened the electrostatic force. The distance between the two charges is denoted by $r$. Choosing a Cartesian system of coordinates in such a way that the surface coincides with the $y, z$ plane the potential of the total force acting on a repeating unit is found by integrating over the whole surface:

$$
V(x)=\int \mathrm{d}^{2} V=(2 \pi q \sigma / \epsilon k) \exp (-k x) .
$$

The case of adsorption of course corresponds to negative values of $q \sigma$. The energy of the electrostatic interactions between the repeating units will be negligible with respect to (2) provided:

$$
q \ll \sigma l^{2},
$$

where $l$ denotes the linear dimension of a repeating unit. In the model under consideration we assume this order of magnitude estimation to hold.

The molecule will be represented by $N$ freely hinged links, each of length $l$, with end points at $\boldsymbol{r}_{0}, \boldsymbol{r}_{1}, \ldots \boldsymbol{r}_{N}$. The configuration sum for a molecule with both end points fixed equals:

$\boldsymbol{G}\left(\boldsymbol{r}_{N}, N \mid \boldsymbol{r}_{0}, 0\right)=\int \mathrm{d}^{3} \boldsymbol{r}_{1} \int \mathrm{d}^{3} \boldsymbol{r}_{2} \ldots \int \mathrm{d}^{3} \boldsymbol{r}_{N-1} \prod_{i=0}^{N-1}\left(4 \pi l^{2}\right)^{-1} \boldsymbol{\delta}\left(\left|\boldsymbol{r}_{i+1}-\boldsymbol{r}_{i}\right|-l\right) \exp \left(-\beta V\left(\boldsymbol{r}_{i+1}\right)\right)$.

Here $\beta=\left(k_{\mathrm{B}} T\right)^{-1}$ with $k_{\mathrm{B}}$ denoting Boltzmann's constant and $T$ the absolute temperature. By first performing the integrations over $\boldsymbol{r}_{1}, \boldsymbol{r}_{2} \ldots \boldsymbol{r}_{N-2}$ at fixed $\boldsymbol{r}_{N-1}$ one finds the integral equation

$G\left(\boldsymbol{r}_{N}, N \mid \boldsymbol{r}_{0}, 0\right)=\left(4 \pi l^{2}\right)^{-1} \int \delta\left(\left|\boldsymbol{r}_{N}-\boldsymbol{r}_{N-1}\right|-l\right) \exp \left(-\beta V\left(\boldsymbol{r}_{N}\right)\right) G\left(\boldsymbol{r}_{N-1}, N-1 \mid \boldsymbol{r}_{0}, 0\right) \mathrm{d}^{3} \boldsymbol{r}_{N-1}$.

For $N \gg 1$ the dependence of $G$ on $N$ will be smooth, so that $N$ can be treated as a continuous variable. By expanding $G\left(r_{N-1}, N-1 \mid r_{0}, 0\right)$ in a Taylor series around the point $\left(\boldsymbol{r}_{N}, N\right)$ it is found that the integral equation can be replaced by the differential equation

$$
\left(\frac{\partial}{\partial N}-\frac{l^{2}}{6} \Delta_{r_{N}}+\mathrm{e}^{+\beta V\left(r_{N}\right)}-1\right) G\left(r_{N}, N \mid r_{0}, 0\right)=0,
$$

where it was assumed that the distance over which $G$ changes its value appreciably is very large as compared to the linear dimension of a repeating unit. For a weakly bound macromolecule one has:

$$
\beta V \ll 1,
$$


and (6) simplifies to

$$
\left(\frac{\partial}{\partial N}-\frac{l^{2}}{6} \Delta_{r_{N}}+\beta V\left(r_{N}\right)\right) G\left(r_{N}, N \mid r_{0}, 0\right)=0 .
$$

This equation has to be solved under the boundary conditions that $G$ vanishes if $\boldsymbol{r}_{N}$ approaches either infinity or a hard wall, and under the initial condition

$$
\lim _{N \downarrow 0} G\left(r_{N}, N \mid r_{0}, 0\right)=\delta\left(r_{N}-r_{0}\right) .
$$

This implies that $G$ is the Green function of the differential operator on the left-hand side of (8).

When the explicit form (2) of the potential is substituted into (8) it is found that the equation separates. Hence $G$ is the product of three functions, each of one variable only. Two of them have the form of the Green function of a one-dimensional free-diffusion equation, and one obeys a non-trivial equation. Using the bilinear expansion of a Green function in terms of the eigenfunctions of the corresponding differential operator one finds:

$$
\begin{aligned}
G\left(r_{N}, N \mid r_{0}, 0\right) & =\left(\frac{2}{3} \pi N l^{2}\right)^{-1} \exp \left\{-3\left[\left(y_{N}-y_{0}\right)^{2}+\left(z_{N}-z_{0}\right)^{2}\right]\left(2 N l^{2}\right)^{-1}\right\} \\
& \times \sum_{n} \phi_{n}\left(x_{N}\right) \phi_{n}^{*}\left(x_{0}\right) \mathrm{e}^{-\lambda_{n} N}
\end{aligned}
$$

where

$$
\left(-\frac{l^{2}}{6} \frac{\mathrm{d}^{2}}{\mathrm{~d} x^{2}}-\theta \mathrm{e}^{-k x}\right) \phi_{n}(x)=\lambda_{n} \phi_{n}(x)
$$

The eigenfunctions are assumed to be orthogonal. The boundary conditions are that $\phi_{n}(x)$ vanishes on the membrane at $x=0$ and for some large positive value $(L)$ of $x$ which corresponds to the other end of the cell:

$$
\phi_{n}(0)=\phi_{n}(L)=0 \text {. }
$$

The length $L$ will of course be very large as compared to the two other fundamental lengths $l$ and $k^{-1}$ in this problem, and can in many cases be put equal to infinity. In (11) we also introduced the adsorption energy of a monomer at the membrane surface in units of $k_{\mathrm{B}} T$ :

$$
\theta \equiv 2 \pi|\sigma q| / k_{\mathrm{B}} T k \epsilon \text {. }
$$

When the differential equation is transformed to the new variables

$$
S \equiv(k l)^{-1}(24 \theta)^{1 / 2} \exp \left(-\frac{1}{2} k x\right) ; \quad f(S) \equiv \phi(x)
$$

its form simplifies to

$$
\left(\frac{\mathrm{d}^{2}}{\mathrm{~d} S^{2}}+\frac{1}{S} \frac{\mathrm{d}}{\mathrm{d} S}+1+\frac{\Lambda}{S^{2}}\right) f(S)=0 ; \quad \Lambda=\frac{24 \lambda}{l^{2} k^{2}}
$$

and the boundary conditions become:

$$
f\left(k^{-1} l^{-1}(24 \theta)^{1 / 2}\right)=f\left(k^{-1} l^{-1}(24 \theta)^{1 / 2} \exp \left(-\frac{1}{2} k L\right)\right)=0 .
$$

The eigenvalues $\Lambda$ of this equation can be negative or positive; both cases will be considered separately. 
For negative values of $\Lambda$ one has $\Lambda=-\nu^{2}$, with $\nu>0$. This transforms (15) into Bessel's equation. The eigenfunctions are bound states which decay exponentially at large positive values of $x$. They have the form

$$
f(S)=A J_{\nu}(S)+B J_{-\nu}(S) .
$$

The boundary conditions (16) lead to the eigenvalue equation:

$$
J_{\nu}\left(S_{1}\right) J_{-\nu}\left(S_{2}\right)=J_{\nu}\left(S_{2}\right) J_{-\nu}\left(S_{1}\right)
$$

with:

$$
S_{1}=S_{2} \exp \left(-\frac{1}{2} k L\right), \quad S_{2}=k^{-1} l^{-1}(24 \theta)^{1 / 2} .
$$

We shall study the solutions of the eigenvalue equation only in the limit $L \rightarrow \infty$ (for a finite value of $L$ the analysis of the spectrum is much more complicated, but as in most realistic cases the length $L$ will be very large compared to $k^{-1}$ there is no need to consider this case). For $S_{1} \downarrow 0$ one can use the limiting form of the Bessel functions for small arguments (Abramowitz and Stegun 1970, equation (9.1.7)):

$$
\begin{array}{ll}
J_{\nu}\left(S_{1}\right) \cong\left(\frac{1}{2} S_{1}\right)^{\nu} / \Gamma(\nu+1) & (L \rightarrow \infty), \\
J_{-\nu}\left(S_{1}\right) \cong\left(\frac{1}{2} S_{1}\right)^{-\nu} / \Gamma(1-\nu) & (L \rightarrow \infty) .
\end{array}
$$

Substituting into $(18 a)$ one finds that one has to solve

$$
J_{\nu}\left(S_{2}\right) \cong J_{-\nu}\left(S_{2}\right) \frac{\Gamma(1-\nu)}{\Gamma(\nu+1)}\left[k^{-1} l^{-1}(6 \theta)^{1 / 2}\right]^{2 \nu} \exp (-k L \nu) ; \quad(L \rightarrow \infty) .
$$

In the limit $L \rightarrow \infty$ the right-hand side goes to zero exponentially for any fixed positive value of $\nu$. Hence in that limit one has to solve the eigenvalues from:

$$
J_{\nu}\left[k^{-1} l^{-1}(24 \theta)^{1 / 2}\right]=0 .
$$

There will be as many bound states as there are positive real values of $\nu$ such that the last equation holds. The ground state of the original eigenvalue problem (11) corresponds to the root of (19) with the largest value of $\nu$. This remark enables us to infer the existence of a phase transition in this problem as follows. It is well known (see Watson 1922 , $\S 15.6$, Jahnke and Emde 1945, figure 84) that the first positive root $j_{\nu, 1}$ of the equation $j_{\nu}(x)=0$ increases with increasing $\nu$. Therefore a phase transition occurs when the dimensionless parameter $k^{-1} l^{-1}(24 \theta)^{1 / 2}$ has the special value

$$
k^{-1} l^{-1}\left(24 \theta_{c}\right)^{1 / 2}=j_{0,1}=2 \cdot 4048 \ldots
$$

For $\theta<\theta_{c}$ equation (19) has no solutions; for $\theta>\theta_{c}$ this equation has at least one solution. The temperature $\left(T_{\mathrm{c}}\right)$ of the phase transition is found by substitution of (13) into the last equation:

$$
k_{\mathrm{B}} T_{\mathrm{c}}=48 \pi|\sigma q| / j_{0,1}^{2} k^{3} l^{2} \epsilon .
$$

The phase transition is such that for $T<T_{\mathrm{c}}$ at least one negative eigenvalue $\lambda_{0}$ exists whereas for $T>T_{\mathrm{c}}$ all $\lambda_{n}$ are positive. Hence for temperatures below the transition temperature the sum over the eigenfunctions in (10) will, for large vlalues of $N$, be dominated by the ground state:

$$
\sum_{n} \phi_{n}\left(x_{n}\right) \phi_{n}\left(x_{0}\right) \mathrm{e}^{-\lambda_{n} N} \simeq \phi_{0}\left(x_{N}\right) \phi_{0}^{*}\left(x_{0}\right) \mathrm{e}^{-\lambda_{0} N} ; \quad(N \gg 1) .
$$


The ground state itself follows by substitution of (14) into (17):

$$
\phi_{0}(x)=A_{0} J_{\nu_{0}}\left[k^{-1} l^{-1}(24 \theta)^{1 / 2} \exp \left(-\frac{1}{2} k x\right)\right] .
$$

The probability density to find the end point of the macromolecule at position $x$ can be found by substituting (21) into (10) and integrating over $y_{N}$ and $z_{N}$ :

$$
P(x, N)=\phi_{0}(x)\left(\int_{0}^{\infty} \phi_{0}(x) \mathrm{d} x\right) ; \quad(N \gg 1) .
$$

This function does not depend on $N$ and is sharply peaked in a narrow layer of thickness of the order $k^{-1}$ near to the membrane. Hence for $T<T_{\mathrm{c}}$ the attractive forces are strong enough to overcome the heat motion and the macromolecule is effectively adsorbed to the membrane.

For $T>T_{\mathrm{c}}$ no bound states exist and all $\phi_{n}$ are positive. The corresponding eigenfunctions have the form of plane waves:

$$
\phi_{n}(x)=C_{n} \sin (x / l)\left(6 \lambda_{n}\right)^{1 / 2}+D_{n} \cos (x / l)\left(6 \lambda_{n}\right)^{1 / 2}, \quad\left(x \gg k^{-1}\right)
$$

provided $x$ is far removed from the membrane. Hence the probability density of the end point will not be localized near the membrane but will, for $N \gg 1$, be effectively constant: $P(x, N)=L^{-1}$. From a physical point of view this means that for $T>T_{\mathrm{c}}$ the heat motion is strong enough to overcome the attractive forces and to shuttle the molecule throughout the cell.

\section{Concluding remarks}

The adsorption behaviour of this model with long-range forces is qualitatively similar to the behaviour in Rubin's models with short-range forces (Rubin 1965). Hence we can safely conclude that this type of behaviour is universal.

In the living cell the phase transition in the adsorption behaviour is not induced by a change of temperature, but by a change in one or several of the variables $\sigma, q, k, \epsilon$, which according to (20) determine $T_{\mathrm{c}}$. The values of these variables depend on the ionic composition of the cell fluid and on the structure of the membrane, i.e. on the details of the cell's metabolism. A slight change in the chemical inventory of the cell can push the system through its phase transition, thereby driving the macromolecule towards (or away from) the membrane. The biological activity of some biopolymers can be quite different, depending on whether the molecule is squeezed near to a membrane or floats feely in the bulk of the cell fluid (Di Marzio and Bishop 1974). In this way phase transitions of the type discussed in this paper could play a role in the regulation of cellular metabolism.

\section{References}

Abramowitz M and Stegun I A 1970 Handbook of Mathematical Functions (New York: Dover)

Di Marzio E A and Bishop M 1974 Biopolymers 132331

Jahnke E and Emde F 1945 Tables of Functions (New York: Dover)

Rubin R J 1965 J. Chem. Phys. 432392

Watson G N 1922 Theory of Bessel Functions (Cambridge: Cambridge University Press) 